\title{
Una iniciativa innovadora para estudiantes de posgrado
}

An innovative initiative for postgraduate students

\author{
Volumen 19, Número 1 \\ Enero-Abril \\ pp. 1-24
}

Este número se publica el 1 de enero de 2019

DOI: https://doi.org/10.15517/aie.v19i1.35060

\author{
Maribel Figueroa Rodríguez \\ Lorna D. Torres López
}

Revista indizada en REDALYC, SCIELO

Revista distribuida en las bases de datos:

\section{LATINDEX, DOAJ, REDIB, IRESIE, CLASE, DIALNET, SHERPA/ROMEO, QUALIS-CAPES, MIAR}

Revista registrada en los directorios:

ULRICH'S, REDIE, RINACE, OEI, MAESTROTECA, PREAL, CLACSO 


\title{
Una iniciativa innovadora para estudiantes de posgrado
}

\author{
An innovative initiative for postgraduate students
}

\section{Maribel Figueroa Rodríguez ${ }^{1}$ \\ Lorna D. Torres López ${ }^{2}$}

Resumen: Múltiples autores y autoras han señalado la falta de servicios dirigidos al estudiantado de posgrado dentro de las instituciones de enseñanza universitaria. Frente a ello, recomiendan que se desarrollen programas en las universidades que atiendan las necesidades de este cuerpo estudiantil. Como respuesta a esta situación, en este ensayo presentaremos la implantación de un proyecto en una universidad privada en Puerto Rico, entre 2010-2015, sus componentes y aportaciones encaminados al fortalecimiento de los programas de posgrado. La experiencia narrada puede servir de guía para personas o miembros de universidades interesados en implantar iniciativas parecidas, con el fin de atender las necesidades del estudiantado de posgrado. Para contribuir a ese fin, incluimos aprendizajes, retos, y concluimos con recomendaciones. Destacamos la imperiosa necesidad de que las universidades se comprometan a atender las necesidades de las personas estudiantes de posgrado a través de actividades y servicios especializados, al igual que apoyos económicos para contribuir a que completen sus grados satisfactoriamente.

Palabras clave: estudiante de posgrado, apoyo pedagógico, enseñanza superior, investigación, docentes.

Abstract: Multiple authors have pointed out the lack of services for postgraduate students in higher education institutions, and recommend the universities to develop programs for addressing the needs of this population. As a response to this situation, in this essay, we describe the implementation of a project with this same goal from a private institution of higher education in Puerto Rico, within 2010 and 2015, as well as, the components and contributions addressed to strengthen the postgraduate programs. The narrated experience here, can guide individuals or members of higher education institutions who are interested on similar initiatives to fulfill the needs of postgraduate students. As a contribution to this purpose, learning challenges and a conclusion with recommendations is included. We highlight the imperious need for the universities to assume this commitment, and, to address the postgraduate students needs by offering them specialized activities and as a result of our experience we offer our reflection of the process and recommendations.

Key words: postgraduate students, student support services, institutions of higher education, research, faculty.

\footnotetext{
1 Universidad del Este, Puerto Rico. Decana Asociada, Decanato de Estudios Graduados. Dirección electrónica: mafigueroa@suagm.edu

2 Universidad del Este, Puerto Rico. Directora, Decanato de Estudios Graduados. Dirección electrónica: lotorres@suagm.edu
}

Ensayo recibido: 3 de mayo, 2018

Enviado a corrección: 8 de agosto, 2018

Aprobado: 17 de setiembre, 2018 


\section{Introducción}

La educación superior está plagada de desafíos y desigualdades. En este caso, abordaremos las desigualdades que confronta el estudiantado graduado ${ }^{3}$ debido a la ausencia de servicios especializados para responder a las necesidades que surgen de sus características y demografía. Comenzaremos con una revisión de literatura sobre la escasez de servicios especializados dirigidos al estudiantado graduado y describiremos cómo el proyecto que implantamos ofreció alternativas. Luego, contextualizaremos su desarrollo dentro de la experiencia de la educación superior en Puerto Rico y, en particular, en la universidad donde se generó. Para ello, presentaremos cada uno de los componentes de este proyecto en su fase de implantación junto con las aportaciones, aprendizajes y retos. Finalizaremos con recomendaciones que esperamos sean útiles para personas interesadas en este tema.

\section{Situación del estudiantado graduado en las Instituciones de Educación Superior (IES)}

Es indiscutible que actualmente las Instituciones de Educación Superior (IES) juegan un rol fundamental en la formación y transformación de personas y sociedades. Tanto sus ofrecimientos académicos como sus servicios son vitales para el desarrollo integral del estudiantado, su retención y graduación. No obstante, al indagar sobre qué tipo de servicios se ofrecen y para quién, en la literatura estadounidense se informa que los servicios estudiantiles de las IES se enfocan en satisfacer principalmente las necesidades del estudiantado de pregrado (Guentzel y Elkins, 2006; Pontius y Harper, 2006; Tacke, 2005). Este dato pone de manifiesto la posición de desventaja en la que se encuentra el estudiantado de posgrado y la inequidad en cuanto a la cantidad y disponibilidad de servicios que consideren sus necesidades y particularidades (Maxey, 2014).

Para Rizzolo, De Forest, De Cino, Strear y Landram (2016), la población estudiantil graduada tiene que competir con una cultura de servicio abrumadora dirigida al estudiantado de pregrado y, además, con personal adiestrado específicamente para esa población. Estos

\footnotetext{
${ }^{3}$ Entiéndase que el término graduado en Puerto Rico, y en los Estados Unidos de América, es el equivalente a posgrado en los países de América Latina. De igual forma, el concepto subgraduado, se debe entender como pregrado.
} 
autores y autoras critican que los recursos para el grupo graduado son limitados y recomiendan que los que se ofrezcan se alineen a sus necesidades particulares.

Wendler, Bridgeman, Cline, Millet, Rock, Bell y McAllister (2010) señalaron que la matrícula del estudiantado, a nivel graduado, va en aumento en Estados Unidos. Esto también ocurre en Puerto Rico y, especialmente, en la Universidad del Este, institución donde se implantó el proyecto que describiremos. De acuerdo con el Sistema Universitario Ana G. Méndez, Universidad del Este (2017), la matrícula graduada aumentó en un 16,5 por ciento (1 281), comparada con el año anterior que fue 1099 estudiantes. A pesar del incremento de la matrícula de estudiantes de posgrado, Guentzel y Elkins (2006) señalaron que, al no ser numéricamente superior al sector subgraduado, se convierten en una población secundaria. Plantea Maxey (2014) que las IES no pueden darse el lujo de ignorar las necesidades de la población de estudiantes de posgrado, o asumir que no necesitan asistencia, ya que son parte de su matrícula.

Ante este panorama la pregunta obligada es ¿por qué los servicios se focalizan en el estudiantado de nivel subgraduado? Fischer y Zigmond (1998, citado en Pontius y Harper, 2006) expusieron cuatro factores: (1) la matrícula subgraduada casi siempre excede numéricamente a la graduada; (2) se cree que la población estudiantil subgraduada requiere más atención y recursos; (3) se piensa que los departamentos académicos satisfacen las necesidades de la población graduada y, (4) se presume que el estudiantado del sector graduado, al poseer un grado académico, sabe lidiar con procesos burocráticos de las IES. Otra de las razones para justificar la falta de servicios al grupo graduado es la falta de recursos institucionales (Terry, 2002).

Por lo antes descrito es necesario visibilizar a la población graduada y, sobre todo, identificar su perfil y sus necesidades para ofrecerle servicios de apoyo que redunden en su desarrollo académico, profesional y personal. Estos servicios también incidirán en las tasas de retención y graduación (Pontius y Harper, 2006). Según Skouras (2001), debido al aumento de la población estudiantil graduada, adulta y no tradicional, las IES deben proveer una amplia variedad de servicios de apoyo. Plantea este autor que es imperativo reconocer que ya no es válido el modelo educativo uniforme (one size fits all) que rige la cultura institucional de servicios de una IES. Un modelo de servicio uniforme, desde una mirada subgraduada, no se ajusta al perfil demográfico y social de la población graduada. 
Sostiene Polson (2003) que la demografía del estudiantado graduado ha cambiado y el cambio no se refleja en la composición de los programas que le ofrecen servicios. Esta autora señala que esta población trabaja a tiempo completo, se matricula a tarea parcial y en muchas instancias tienen que viajar largas distancias para llegar al campus. Añade Tacke (2005) que el perfil de esta población se distingue por el aumento de personas adultas con familia, por ser mayoritariamente mujeres y ser de otros países. Otras características del estudiantado graduado son la gran cantidad de responsabilidades que asumen fuera del salón de clases y la falta de tiempo para estar más horas, físicamente, en la universidad (Maxey, 2014). Peterson (2006) expuso que en la matrícula graduada se ha registrado un aumento de personas con algún reto físico o cognitivo, con problemas de aprendizaje y con la responsabilidad primaria de cuidar una persona menor o adulta. Estas variables representan un reto para los estudiantes al tratar de balancear los estudios y su vida personal.

Para Bailey-Chen (2007), entender estos cambios es vital para que las IES planifiquen y mejoren sus servicios. El diverso y complejo perfil del estudiantado graduado le requiere a las IES diseñar programas y servicios centrados en esta población mientras se cumplen las metas y prioridades de la institución (Baily-Chen, 2007; Elkins, Guentzel, Gansemer-Topf, Ewing y Turrentine, 2006). A continuación, abordaremos los servicios recomendados para este sector estudiantil, los cuales surgen de un resumen de investigaciones, iniciativas y descripción de programas para esta población.

\section{Servicios y programas para el estudiantado de posgrado}

Una investigación realizada por Rizzollo, DeForest, DeCino, Strear y Landram (2016), en EE. UU., tuvo como objetivos evaluar el desarrollo de un programa de desarrollo profesional para estudiantes de posgrado y explorar las percepciones y experiencias acerca de este tipo de actividades. Participaron 689 estudiantes que completaron un cuestionario y 13 estudiantes que participaron en un grupo focal. En resumen, las personas participantes expresaron que las actividades de desarrollo profesional son necesarias para el estudiantado graduado. Entre las actividades, mencionaron las relacionadas con la preparación para la carrera, tales como talleres de preparación de resumé, curriculum vitae y entrevistas de empleo. Se les preguntó acerca de las barreras para asistir a las actividades y mencionaron la falta de tiempo y de recursos económicos. Los investigadores e investigadoras 
recomendaron que los programas de desarrollo profesional incluyan educación continua, investigación y actividades con pares y personas mentoras.

De otro lado, se plantea que los programas de orientación son fundamentales para apoyar al estudiantado graduado en transiciones académicas y sociales (Polson, 2003). Una actividad de orientación, al ingresar al programa, es vital para presentar recursos institucionales más allá de los que proveen los departamentos académicos (Pontius y Harper, 2006). Otras áreas identificadas para el desarrollo de servicios al estudiantado graduado son la asesoría, programas de ayudas económicas y la creación de centros o espacios para el uso de este sector (Guentzel y Elkins, 2006; Maxey, 2014; Polson, 2003).

La creación de centros para uso de la población graduada es una recomendación frecuente (Brandes, 2006; Maxey, 2014; Polson, 2003). Brandes (2006) explicó que los centros para esta población deben incluir espacios, servicios, programas especializados y personal de servicios estudiantiles. Los edificios construidos para este propósito deben contar con salones de estudio, áreas de socialización, salas de conferencias, laboratorios de computadoras con impresoras, muebles cómodos, tablones de edicto para colocar avisos, acceso a internet y oficinas del personal de servicio. Esta autora esboza varias razones para enfatizar la importancia de estos centros, entre ellos: promueven el sentido de comunidad entre el estudiantado, le dan visibilidad a la población graduada y transmiten el mensaje de que la institución valora y reconoce a ese sector.

No obstante, un espacio físico en sí mismo es insuficiente. Es necesario que en el centro se fomente la interacción entre pares, y entre estudiantes y docentes (Brandes, 2006). La autora recomienda que en estos centros se ofrezcan servicios, actividades académicas, sociales y de desarrollo profesional, y que además, se coordinen servicios en colaboración con otras oficinas de la institución. Otra área de servicio fundamental para la población estudiantil graduada es la de ayudas económicas para sufragar los costos de estudio. En un estudio de necesidades de estudiantes de posgrado en EE.UU., Skouras (2001) reportó que una de las necesidades más apremiantes del estudiantado participante fue la falta de becas o ayudas económicas.

Dado que las ayudas económicas son limitadas, financiar los estudios es una gran preocupación para este sector y un factor determinante en su decisión de matricularse y permanecer en la institución (El-Khawas, 2003). En este sentido, los cambios más dramáticos que están ocurriendo en la educación superior son las numerosas solicitudes de 
préstamos y, como consecuencia, el aumento en la deuda estudiantil (Patel, 2014). Según El-Khawas (2003), un gran número de estudiantes del sector graduado son los proveedores principales de sus familias y, como resultado, esta realidad les produce estrés ya que deben renegociar sus finanzas familiares para costear sus estudios.

En la literatura antes expuesta se recalca la falta de servicios dirigidos al estudiantado graduado y la urgencia de que las universidades desarrollen programas que atiendan sus necesidades. Ante esto, a continuación, describiremos el proyecto que se implantó en la Universidad del Este de Puerto Rico considerando el contexto de las IES y el perfil del estudiantado graduado de la Isla.

\section{La Universidad del Este: Escenario de esta experiencia}

Las transformaciones sociales, económicas y demográficas han impactado a las IES en Puerto Rico de forma directa. Estos cambios han incidido en la matrícula, el perfil del estudiantado universitario y la contratación de docentes (Aponte, Báez, Rivera y Calderón, 2015; Rosario, 2014). El Consejo de Educación de Puerto Rico informó que a julio de 2016 operaban en el país 59 IES. De estas, 55 pertenecían al sector privado. Para el año académico 2016-2017, un 13\% de la matrícula de las IES correspondía al nivel graduado (Rivera y Calderón, 2017). Tanto Alicea (2012) como Aponte et al. (2015), señalaron que las IES en Puerto Rico confrontan serios retos debido a factores como: a) la emigración; b) la disminución poblacional; c) el alto nivel de desempleo; d) el aumento de familias no tradicionales y e) la dependencia de fondos federales de los EE.UU. para sufragar los costos de matrícula. Las restricciones de tiempo del estudiantado que trabaja a tiempo parcial o completo es otro de los retos. Al examinar los retos antes descritos, no es casualidad que se reflejen en la IES en la que se implantó el proyecto y en el perfil de su población graduada.

La Universidad del Este (UNE) es una de las IES privadas en el país y es parte del Sistema Universitario Ana G. Méndez (SUAGM) junto a la Universidad Metropolitana, Universidad del Turabo y Universidad Virtual. EI SUAGM cuenta con centros universitarios alrededor de la Isla, y en cuatro estados de los EE.UU. Estos incluyen Florida, Maryland, Texas y Washington. EI SUAGM es actualmente la IES más grande en Puerto Rico, con una matrícula de 43,284 estudiantes ("Puerto Rico's largest universities", 2017). Esto nos sugiere el impacto que puede tener en la formación académica del estudiantado universitario del 
país, en la planta docente que la integra y en el desarrollo laboral y económico de Puerto Rico.

La UNE, en particular, tiene una matrícula total de 13, 058 estudiantes. En el recinto de Carolina se agrupan 5,605. (UNE, 2016). Para propósitos de este artículo nos referiremos exclusivamente al campus de Carolina y a sus programas graduados, áreas en las que se enfocó el proyecto que describiremos. La UNE sirve a los municipios del área noreste de Puerto Rico. De acuerdo con el Censo de 2010, en esta área geográfica de la Isla, reside una población con gran necesidad económica (Departamento de Comercio de los EE. UU., 2013). El estudiantado que ingresa a la Institución procedente de esta realidad socioeconómica constituye un gran reto debido a las circunstancias académicas y de vida que les acompañan.

\subsection{Contexto de los programas graduados de la UNE}

Los primeros programas graduados comenzaron en el año 2001 mediante la aprobación del Consejo de Educación Superior (CES) (posteriormente, Consejo de Educación de Puerto Rico), agencia acreditadora nacional. Inicialmente hubo dos maestrías adscritas a la Escuela de Educación y en el 2003 se graduaron los primeros ocho estudiantes de esos programas (Universidad del Este, 2009). La institución continuó diversificando su ofrecimiento académico a partir del 2001 y, como resultado, aumentaron los programas graduados a nivel de maestría y las especialidades hasta las existentes en la actualidad. Los programas graduados se agrupan en las Escuelas de Ciencias Sociales y Humanas, Educación, Innovación, Emprendimiento y Negocios, y en la Escuela de Estudios Profesionales. Los cursos se ofrecen principalmente en el término académico conocido como Part of Term (ocho o cinco sesiones de tres horas a la semana) con excepción del Programa de Trabajo Social Clínico, cuyo ofrecimiento académico se da en semestres. El horario de los cursos es vespertino, de lunes a viernes, y diurno los fines de semana (sábado y domingo).

A pesar de las múltiples funciones estudiantiles que los programas graduados continuaron ejerciendo, la dirección de las Escuelas enfatizó que el estudiantado carecía de servicios y apoyos adicionales que contribuyeran a su éxito académico. Esta carencia estaba sustentada con datos de diversos estudios, como: la evaluación realizada por la Vicerrectoría de Asuntos Estudiantiles, cuestionarios de fin de curso, cuestionarios de satisfacción de servicios a estudiantes y entrevistas a docentes y estudiantes. Además, se evidenciaba con 
análisis de otros datos, entre ellos: matrícula graduada, índice de graduación y retención. Con esta recopilación de información se sustentaron las principales necesidades de fortalecer los servicios académicos y de apoyo al estudiantado graduado, aumentar las instalaciones físicas e infraestructura tecnológica, integrar a docentes a tarea completa y parcial, y ofrecerle mayor apoyo económico al estudiantado.

\subsection{Perfil de la población estudiantil graduada de la UNE}

En una encuesta sobre este sector (UNE, 2015) se reportó que: a) la mayoría son mujeres (70\%); b) tienen un promedio de edad de 34 años; c) el $40 \%$ reside en el municipio de Carolina; d) la mayoría trabaja (86\%) y, e) el $82 \%$ tiene préstamos estudiantiles del gobierno federal de los EE.UU. Este perfil es similar al que se reporta en la literatura estadounidense (Bailey-Chen, 2007; Polson, 2003). El perfil antes descrito y las necesidades identificadas sirvieron de base para el proyecto que describiremos a continuación.

\section{Componentes del Proyecto Título V Graduado}

\subsection{Estructura organizacional}

Este proyecto fue financiado por el Departamento de Educación de los EE.UU. por un periodo de cinco años (2010-2015). La estructura del proyecto incluyó personal administrativo, dos comités asesores, docentes y estudiantes adscritos/as a dos de los componentes del proyecto. El personal administrativo incluía: directora, asistente administrativa, coordinadora de servicios integrados, especialista de investigación y coordinadora de servicios administrativos. Para algunos puestos se requería una maestría. No obstante, se reclutaron personas con grado doctoral para los puestos de coordinadora de servicios integrados y la especialista de investigación. Ambas personas tenían vasta experiencia, compromiso y una visión clara sobre el desarrollo de los estudios graduados.

El proyecto recibió asesoramiento Ad Honorem de dos comités: Advisory Board (compuesto por vicerrectores y vicerrectoras de la UNE) y el Oversight Committee (compuesto por representantes del cuerpo docente y estudiantes de cada uno de los programas graduados). Para la evaluación del proyecto se contrató a una evaluadora externa. Al final de cada año, la directora del proyecto rendía un informe a la agencia financiadora y al final de los cinco años sometió una evaluación sumativa. El proceso de evaluación consistió de grupos focales de estudiantes, docentes y personal del proyecto; 
además del análisis de documentación y datos estadísticos. En la siguiente sección explicamos cada uno de los componentes del proyecto, sus objetivos, las actividades realizadas y los resultados principales.

\subsection{Servicios académicos y de apoyo estudiantil}

El objetivo principal de este componente era brindar y coordinar servicios que contribuyeran al desarrollo, la retención y la culminación del grado de maestría. También se gestionaban referidos o servicios de colaboración con otras unidades de la Institución. Los servicios estaban dirigidos a todo el estudiantado, desde su admisión, y eran liderados por la coordinadora de servicios integrados y por estudiantes recipientes de una beca, designados a este componente. Al estudiantado graduado de nuevo ingreso, se le ofrecía una actividad de bienvenida al inicio de cada semestre académico. Esta incluía charlas sobre los servicios de la UNE, del proyecto y recorridos por la Universidad.

Los servicios eran variados y se enfocaban en el desarrollo académico, profesional y personal de la población graduada. Durante dos años (2012 y 2013) se implantó un programa de mentoría de pares. A partir del año académico 2013-2014, se ofrecieron ciclos de talleres de redacción para fortalecer esta destreza. El estudiantado de Trabajo Social Clínico y Justicia Criminal también participó en talleres sobre estrategias de preparación y contestación de exámenes comprensivos (requisito de estos programas). A través de estos talleres el estudiantado recibía, además, estrategias sobre hábitos de estudio. Como parte de las actividades de desarrollo profesional, se coordinaron talleres sobre el uso de diversos programas para preparar hojas de cálculo, elaboración de presentaciones, creación de blogs, entre otros. Además, se ofrecieron adiestramientos sobre preparación de resumé y cómo prepararse para una entrevista de empleo. Asimismo, se coordinaron visitas de compañías que tenían ofertas de empleo disponibles.

El proyecto, a través del componente de servicios, también apoyó el desarrollo personal del estudiantado graduado, ya que este sector lidia con muchas presiones (académicas, personales, económicas) que, en ocasiones, ponen en riesgo su aprovechamiento y desempeño en la sala de clases. Durante los cinco años del proyecto se facilitaron talleres sobre el manejo del estrés, las emociones, la frustración y el fracaso. Además, se ofrecieron talleres de manejo del tiempo, bienestar y destrezas de organización. La mayoría de estos talleres se organizaron en colaboración con la unidad de Consejería de 
la institución. Durante los cinco años del proyecto se atendieron 699 estudiantes en el componente de servicios, con un promedio de tres intervenciones por estudiante.

Como parte del componente de servicios, en el 2012, se fundó la Asociación de Estudiantes Graduados de la UNE (AEG-UNE) cuya mentora era la coordinadora de servicios integrados. Esta organización se creó para agrupar al estudiantado graduado y desarrollar sus oportunidades de liderazgo. La AEG continúa funcionando y se enfoca en el servicio comunitario y en la promoción de la responsabilidad social del movimiento estudiantil. Algunos eventos celebrados fueron: mesas informativas, conversatorios sobre temas sociales, donaciones y visitas a entidades sin fines de lucro.

Desde el componente de servicios, se originó la publicación de un periódico para el estudiantado graduado llamado Momentos, único periódico estudiantil en la UNE. Esta iniciativa comenzó en el 2013 y hasta el 2015 se publicaron cinco volúmenes. En este periódico se incluía un resumen de las actividades del proyecto, entrevistas a estudiantes y personal de la universidad, artículos relacionados con la vida estudiantil y promociones de actividades del proyecto. Esta publicación fue plataforma de adiestramiento para dos estudiantes becadas que fungieron como editoras. Cabe señalar que el estudiantado del componente planificaba, organizaba y, en muchas instancias, facilitaba las actividades.

\subsection{Sala de Adiestramiento en Investigación (SAI)}

El objetivo de construir la SAI fue fortalecer la infraestructura tecnológica y las destrezas de investigación e información del estudiantado graduado. La SAI tiene un diseño innovador de mesas, 26 computadoras, cuatro pantallas LCD y capacidad para transmitir teleconferencias y videoconferencias. Es un espacio construido y diseñado con la tecnología más avanzada, rodeado de un ambiente de aprendizaje estimulante e innovador. Las computadoras tienen programas que apoyan las tareas académicas del estudiantado en distintas áreas como: análisis de datos (por ejemplo, SPSS y N-VIVO), de sistemas de información y de referencias bibliográficas (entre ellos, QGIS y Mendeley). Además, en la SAI, el estudiantado redacta e imprime sus trabajos, entre otras funciones. Este lugar se diseñó tomando en consideración las necesidades y características de la población, incorporando su perspectiva y las recomendaciones de comités asesores, docentes y otras personas y entidades asociadas. 
La persona especialista de investigación tuvo entre sus funciones la coordinación del SAI. Fue la persona encargada de diseñar y coordinar un programa de talleres de investigación e información que estaba disponible durante el año académico. Estos talleres también estuvieron disponibles para el personal docente. Complementando estos talleres, este personal especialista de investigación ofrecía asesoría al estudiantado y a la planta docente en sus trabajos de investigación, presentaciones y publicaciones.

Otra iniciativa que se desarrolló desde la SAI fue la celebración de tres encuentros de investigación graduada, exclusivamente para estudiantes de la UNE. En esta actividad, estudiantes, junto a mentores y mentoras, presentaban trabajos de investigación desarrollados en cursos o propuestas y proyectos diseñados como requisito de grado. Los trabajos sometidos eran evaluados por un comité científico y se presentaban en formato de cartel o presentación oral. Estos encuentros se celebraron en los años 2012, 2014 y 2015. En esos años, se presentaron un total de 24 trabajos, con igual número de docentes que ofrecieron mentoría y con 54 autores y autoras.

\subsection{Iniciativas de investigación}

Este componente tuvo el objetivo de facilitar la redacción de propuestas de investigación del personal docente y someterlas a agencias de financiamiento. Cada año se abría una convocatoria entre el cuerpo docente de la UNE. Se escogían tres docentes y durante un año se les ofrecía un estipendio y la asistencia de un estudiante becado por el proyecto. El especialista de investigación diseñaba un plan de trabajo para cada díada y les ofrecía los apoyos y la asesoría necesaria para el desarrollo de la redacción de la propuesta, incluyendo la identificación del fondo, los adiestramientos en el área de investigación y la presentación ante la comunidad universitaria al final del año. El plan incluía talleres, asesoría individual, coordinación con otras oficinas (como la de Recursos Externos y la Vicerrectoría de Investigación) y reuniones periódicas, la persona docente investigadora, su estudiante con beca asignada y el personal del proyecto. Al final del año, la persona docente debía someter la propuesta ante una agencia de financiamiento y presentarla ante la comunidad universitaria.

Como resultado de este esfuerzo se redactaron 12 propuestas, con lo cual se cumplió con el plan de trabajo establecido. Estas fueron redactadas por 12 docentes con el apoyo de 15 estudiantes en su rol de asistentes de investigación. El número de estudiantes excede el 
número de docentes ya que hubo sustitución de estudiantes en las diadas debido a graduaciones, cambio de empleo, entre otras. El grupo de estudiantes procedía de distintos programas graduados. Once de los doce equipos de investigación (docente y estudiante) presentaron sus propuestas ante la comunidad universitaria y la sometieron a una agencia financiadora. Estas dos actividades no eran parte de la propuesta; sin embargo, fueron añadidas en la fase de implantación del proyecto. Las propuestas sometidas a las agencias de financiamiento recibieron muy buenas críticas, sin embargo, ninguna logró aprobación ni financiamiento. Solo una investigadora re-sometió a otra fuente de financiamiento cuando ya había concluido el proyecto. Este componente representó una oportunidad única para intercambiar experiencias de investigación entre la planta docente, el estudiantado y el personal administrativo de la UNE.

\subsection{Desarrollo del cuerpo docente}

Los objetivos de este componente eran: a) brindar un espacio de encuentro para que la comunidad graduada de la institución intercambiara experiencias y aprendizajes; b) discutir un tema de prioridad institucional que tuviera un impacto dentro del currículo graduado; y c) capacitar a las personas docentes en distintos temas, entre ellos: las mejores prácticas del proceso de enseñanza - aprendizaje, avalúo estudiantil, mentoría y revisiones curriculares.

Este componente fue liderado por la coordinadora de servicios administrativos. Cada año se celebraron uno o dos retiros para facultad graduada, según lo establecido en la propuesta, para un total de seis. La facultad provenía de las cuatro Escuelas que tienen programas graduados en el recinto de Carolina y los cinco centros universitarios. En el retiro, se ofrecía una plenaria en la mañana, que abordaba un tema institucional y en la tarde se ofrecían distintos talleres por Escuela.

La actividad de la mañana se concentró en sesiones grupales de trabajo dedicadas al tema de avalúo. Este trabajo estuvo liderado por el Vicerrector Asociado de Avalúo de la UNE. Las personas participantes se involucraron de manera intensa y entusiasmada en las discusiones grupales por Escuela, entre ellas: revisión de criterios de rúbrica para medir una competencia, definición de criterios para las rúbricas y revisión de instrumentos para evaluar el aprendizaje estudiantil. Al final, se resumían las discusiones grupales y se informaban al resto de las personas presentes. Estas actividades contribuyeron a desarrollar e implantar 
los planes de avalúo en los programas graduados mediante la construcción e implantación de 15 rúbricas institucionales.

La selección de los temas y los recursos de los talleres por Escuela se identificaban en conjunto con los decanos o directores de programa. Se pautaban varias reuniones con una diversidad de propósitos, entre ellos: a) identificar el tema y el recurso; b) discutir el borrador del taller, c) orientar al recurso sobre la población a atender y sobre otros detalles de logística. Este trabajo cumplió el propósito de asegurarnos que la actividad planificada satisficiera las necesidades de la facultad y el producto resultara pertinente e interesante. Se coordinaron un total de 25 talleres en los retiros, distribuidos en los siguientes temas: enseñanza (12); investigación (4); publicaciones (3); mentoría (3); internacionalización (1); aprendizaje en servicio (1) y reválida (1). Se evaluó la satisfacción del personal docente en todos los retiros y el nivel de satisfacción fue sobre 90 de 100.

\subsection{Becas}

A través de este componente se otorgaron becas anuales por la cantidad de $\$ 6,000$ a estudiantes que respondieron a una convocatoria y pasaron por un riguroso proceso de selección. Cada solicitante debía cumplir con requisitos relacionados con necesidad económica, aprovechamiento académico y disponibilidad de tiempo. Además, se les solicitaba un ensayo justificando la selección del componente de su interés y les entrevistaba un comité de selección, integrado por personal del proyecto y docentes, según el componente deseado.

Cada año fueron seleccionados, por el comité de selección, seis estudiantes graduados (tres en el componente de servicios y tres en iniciativas de investigación). Cada estudiante debía cumplir con 15 horas semanales de trabajo en el componente al que estaba asignado. Según el componente, cada estudiante era supervisado por la persona docenteinvestigadora o por la coordinadora de servicios integrados. Durante su trayectoria, el proyecto otorgó becas a un total de 29 estudiantes (14 en el área de servicios y 15 en iniciativas de investigación).

Hemos presentado la implantación de los distintos componentes del proyecto. Ahora mencionaremos las aportaciones más significativas del proyecto al fortalecimiento de los programas graduados. Estas se presentan agrupadas por categorías. 


\section{Aportaciones}

\subsection{Fortalecimiento de destrezas de información e investigación}

Varias actividades realizadas en el proyecto contribuyeron al fortalecimiento de esta destreza, incluyendo los talleres de información e investigación, los encuentros de investigación, las iniciativas de investigación y los retiros de facultad. El aprendizaje que el estudiantado y personal docente obtuvo, a través de las distintas experiencias, y su nivel de satisfacción, lo pudimos recoger mediante el proceso de evaluación del proyecto (Universidad del Este, 2015).

Para el desarrollo de esta destreza se requieren intervenciones sistematizadas, consistentes e integradas por un periodo de tiempo prolongado, con lo que el proyecto cumplió a lo largo de sus cinco años. Otros factores que contribuyeron fueron el espacio físico existente y los recursos tecnológicos disponibles para ofrecer estos talleres, en este caso la SAI y el personal especializado destacado para atenderlo, como lo fue el especialista de investigación.

Era la primera vez que la población graduada contaba con un programa de talleres enfocado en sus necesidades particulares de investigación con recursos consistentes y disponibles en instalaciones físicas diseñadas para este fin. A lo largo del tiempo, la aceptación hacia los talleres fue aumentando entre el estudiantado. Lo pudimos evidenciar, cuando se duplicó el número de talleres, al comparar el primer año (30) con el último (61). Además, incrementó la diversidad de tópicos. Otro elemento de aceptación fue que uno de los programas graduados estableció, como requisito de graduación para sus estudiantes, la asistencia compulsoria a cuatro de los talleres del programa. Durante la existencia del proyecto se ofrecieron 191 talleres con una asistencia total de 1,666 estudiantes y un nivel de satisfacción de sobre un 93\% durante los cinco años.

Las iniciativas de investigación fueron una experiencia única para el personal docente participante ya que le proveyó un estipendio, una persona asistente de investigación y un plan educativo para desarrollar una propuesta de investigación. Esta situación se aparta de la norma, ya que, usualmente, estos recursos se otorgan cuando se aprueban las propuestas. Sin embargo, en el proyecto, estos recursos estuvieron disponibles para apoyar la fase de redacción de la propuesta. Estas iniciativas, además, permitieron que docentes y estudiantes colaboraran juntos y se expusieran a experiencias de investigación y de mentoría que fortalecieron su desarrollo profesional, según lo expresaron en los grupos 
focales. Igualmente, manifestaron que habían adquirido otras destrezas, entre ellas: destrezas de información, redacción, pensamiento crítico, liderazgo y presentaciones orales.

En el caso de las personas docentes, otro beneficio fue transferir al salón de clases las destrezas y conocimientos obtenidos, según comentaron en los grupos focales. Además, este sector trabajó en el desarrollo de 15 rúbricas para el avalúo a nivel graduado y ese producto se integró a la documentación necesaria para que la Middle States Comission on Higher Education re-acreditara la institución. Por otra parte, la asesoría ofrecida al estudiantado les estimuló, no solo a completar sus requisitos académicos satisfactoriamente, sino a presentar trabajos dentro y fuera del país, e incluso a publicarlos. Fue la primera ocasión en que sobresalieron en actividades investigativas de la UNE y en foros locales e internacionales.

Algunos estudiantes presentaron sus trabajos en foros estudiantiles como la Jornada de la Asociación de Psicología para la Promoción de la Investigación Estudiantil (APPIE) de la Universidad de Puerto Rico, así como en foros profesionales, como la Convención Anual de la Asociación de Psicología de Puerto Rico (APPR). Fuera del país presentaron trabajos en congresos internacionales, entre ellos los Congresos Regionales de la Sociedad Interamericana de Psicología en Bolivia, en El Salvador y en el Congreso Interamericano de Psicología en Perú. Estas presentaciones estudiantiles se convirtieron en artículos publicados en revistas profesionales como la Revista Puertorriqueña de Psicología (Rivera, Toro Alfonso y Meléndez, 2013), Ámbito de Encuentros (Cuevas, Méndez y Benítez, 2014) y en la revista comunitaria Alianza para la Paz Social, ALAPAS (Del Rosario, 2014).

Los encuentros de investigación fueron un logro inesperado, ya que esta actividad se añadió a la fase de implantación del proyecto, pues no estaba incluida en la propuesta aprobada, y se realizó durante tres años. En ellos se expusieron 24 trabajos de investigación, con un total de 54 autores y autoras. Además, fue una oportunidad única e innovadora que le abrió un espacio al estudiantado para exponer sus trabajos de investigación y colaborar con sus mentores/as. En resumen, los estudiantes mencionaron el impacto que habían tenido estas experiencias para adquirir más conocimientos sobre cómo se hace investigación y en ofrecerles un espacio fuera del salón de clases para desarrollar otras destrezas. Esta información se recopiló a través de los distintos instrumentos de evaluación. 


\subsection{Servicios académicos y de apoyo estudiantil}

Desde el componente de servicios, el proyecto ofreció un repertorio de actividades que atendió la diversidad de intereses y necesidades de la población estudiantil graduada. Entre los servicios, cabe destacar los siguientes: orientaciones iniciales, talleres académicos, profesionales y de desarrollo personal (redacción, preparación para el mundo laboral, manejo de estrés, entre otros). Estos servicios concuerdan con los que se recomiendan en la literatura para esta población (Polson, 2003; Rizzollo et al., 2016).

\subsection{Aumentar sentido de identidad y visibilidad del estudiantado graduado}

Sin lugar a dudas, la construcción del edificio del Centro de Desarrollo de Estudios Graduados (conocido con las siglas de CeDEG), brindó un espacio físico, tangible, en el cual el estudiantado podía congregarse e identificarse como comunidad. Una de las aportaciones de este espacio fue visibilizar al estudiantado graduado. Con frecuencia, las personas se referían al edificio y decían: "allí están los graduados o allí está la escuela graduada". En ocasiones, la población posgraduada comentaba que el CeDEG era como un refugio, un espacio de encuentro, conversación y fraternidad con sus pares y con personal del proyecto.

Contar con un lugar donde reunirse o sencillamente estar, le da un sentido de comunidad, de pertenencia, de identidad al estudiantado (Brandes, 2006). El edificio consiste en cinco salones, una sala de conferencia, la SAI, oficinas administrativas y un Learning Commons (sala de estudio). Estas instalaciones representaron un espacio físico que aumentó la cantidad de salones y fortaleció la infraestructura tecnológica, especialmente para el estudiantado graduado. En los grupos focales al estudiantado, se recogió la importancia de este espacio para su sentido de comunidad y desarrollo académico.

\subsection{Fortalecer el desarrollo del personal docente}

Hacer intervenciones efectivas dentro del escenario universitario requiere incluir los diversos sectores que lo componen. Si pretendemos mejorar los servicios al estudiantado graduado es necesario integrar la facultad (Wendler et al., 2010). En el proyecto reconocimos esta necesidad desde sus inicios y el proyecto aportó esta mirada global e integral. Las oportunidades que brindó el proyecto para el desarrollo del personal docente fueron varias. En esta sección, destacaremos las aportaciones de los retiros ofrecidos este personal graduado en su desarrollo profesional. Los retiros no solo constituyeron un intercambio 
académico enriquecedor, sino que permitieron que las personas participantes se relacionaran en un ambiente más personal y relajado, atmósfera que contribuye a fortalecer la comunidad universitaria.

Estas experiencias la ofrecen otras universidades (Rhode Island University, School of Management, y Austin Peay State University, School of Education) con objetivos similares a los del proyecto: aumentar el conocimiento sobre el binomio enseñanza-aprendizaje, compartir ideas, acercamientos o perspectivas innovadoras y promover colaboración e intercambios entre la facultad. Wendler et al., (2010), señalaron que uno de los retos importantes que enfrenta la educación superior es preparar la facultad para manejar los cambios en la educación, particularmente los demográficos, tecnológicos y la manera en que el estudiantado aprende. Otras razones para capacitar a la planta docente es su importante rol en la mentoría y asesoría de estudiantes, y en la consejería sobre carreras profesionales, ya que son los modelos a emular.

En los retiros cumplimos con ofrecer capacitación profesional en temas encaminados a cumplir con esos propósitos. De los 25 talleres ofrecidos, 23 se distribuyeron en los temas de: enseñanza (12), investigación (4), publicaciones (4) y mentoría (3). Otros estudios reconocen las ventajas que ha tenido ofrecer retiros al personal docente en diversos temas. Por un lado, Rosser, Rugg y Ross (2001) apuntan al efecto sinérgico, la alta satisfacción de las personas participantes y la importancia de "retirarse" a un lugar específico para dedicar tiempo y concentración a un tema en particular como ingredientes esenciales para lograr los objetivos. Igual experiencia tuvo el proyecto con los retiros. Igualmente, Haviland, Shin y Turley (2010), quienes ofrecieron talleres sobre actitudes, autoconfianza y entendimiento en relación con los programas de avalúo, señalaron que incluir a las personas docentes en estos procesos tiene un efecto positivo, pues nutre el proceso con entendimiento, confianza y actitudes positivas.

Reconocemos que una facultad más competente, comprometida con la institución y satisfecha, ofrecerá un servicio de más calidad y estimulará entre el estudiantado esas mismas actitudes y compromiso. Un ambiente parecido se logró cuando trabajamos el tema de avalúo con el profesorado. Además, la contribución realizada por el cuerpo docente con el avalúo graduado, fortaleció los procesos de enseñanza-aprendizaje, según expresado por la facultad en los grupos focales realizados como parte del proceso de evaluación. 


\subsection{Ofrecer apoyo económico a estudiantes}

Para El-Khawas, (2003), el factor económico es uno de los principales factores de estrés del estudiantado graduado y un factor decisivo para iniciar y completar estudios de posgrado. Por lo tanto, el otorgamiento de ayudas económicas es un elemento imprescindible, ya que incide en la retención e índice de graduación, además que contribuye a focalizar al estudiantado en sus estudios y no los distrae con presiones económicas (Lvovich, 2009). De 29 estudiantes que se beneficiaron de las becas, un 79\% completó su grado académico. Sin embargo, en la mayoría de los casos, estas ayudas necesitan complementarse con otras fuentes de ingreso, debido a los altos costos de estudio y complicadas situaciones familiares, laborales y personales. Aunque reconocemos su valiosa aportación, se sugiere que se evalúe el impacto de ellas en la formación académica y profesional de la población estudiantil (Luchillo, 2010).

Es importante recalcar que la obtención de la beca representó un beneficio económico, así como también la oportunidad de exponerse a experiencias enriquecedoras. El estudiantado presentó en foros nacionales e internacionales, en jornadas de investigación, organizó actividades académicas y comunitarias, ocupó puestos en asociaciones estudiantiles y obtuvo reconocimientos especiales por sus ejecutorias.

\subsection{Visión del líder y del grupo en la configuración del nuevo escenario}

La narración de esta experiencia no estaría completa sin abordar el rol del personal que trabajó en la implantación del proyecto. Según Sarason (1972) cuando se crea un nuevo escenario, y este fue el caso del proyecto, la visión de la persona líder y del equipo de trabajo, que se congrega para crearlo, hace la diferencia en el carácter que adquiere. La visión del equipo de trabajo del proyecto contribuyó en la forma particular de implementar la propuesta. Como ejemplos de esto podemos mencionar la integración de actividades no consideradas originalmente en ella, las cuales se convirtieron en logros inesperados: los encuentros de investigación, la presentación de las iniciativas ante la comunidad universitaria y ante agencias financiadoras. Otro factor que fortaleció el proyecto fue la poca variación en la composición del personal, elemento importante para el cumplimiento de las metas y para mantener una visión particular de trabajo. Contar con un personal con excelentes credenciales y competencias y, además, con un alto sentido de compromiso, constituyó otro de los pilares en el éxito del trabajo. 
Todo proyecto enfrenta retos y genera aprendizajes. A continuación, los mencionaremos y concluiremos con recomendaciones que pueden servir de referencia para otras personas o miembros de IES interesados en fortalecer los estudios graduados.

\section{Retos}

\subsection{Fomentar la investigación}

Promover la investigación, requiere recursos y apoyos diversos provistos consistentemente a lo largo del tiempo. Esta fue la experiencia del proyecto con los talleres de investigación e información y las iniciativas de investigación. Estudiantes y docentes contaron con un edificio, una infraestructura tecnológica, un personal especializado y apoyos que estuvieron disponibles por espacio de cinco años. Aunque se proveyeron recursos económicos para las personas docentes y el estudiantado del componente de iniciativas de investigación, los recursos se limitaban al año en que participaban del proyecto. Luego de su participación en el proyecto, las condiciones laborales y de recursos para esta población cambiaban y, en muchos casos, las oportunidades para continuar investigando disminuían. Esto es un reto institucional fundamental, principalmente, cuando una de las metas de la Institución es el desarrollo de investigadores e investigadoras.

Reconocemos que un periodo de cinco años es un tiempo corto para robustecer la investigación en la institución. Sin embargo, los esfuerzos realizados fueron pasos en esa dirección. Un mayor despunte en el fortalecimiento de la investigación dependerá de la permanencia y consistencia de estos esfuerzos y de los recursos disponibles.

\subsection{Proceso participativo}

El proceso participativo que se dio junto a decanos y decanas en la planificación de los retiros requirió de grandes esfuerzos e inversión de tiempo debido a sus agendas complicadas. Igual experiencia ocurría cuando se organizaban comités de trabajo integrando la participación de personal de las oficinas de servicio y del personal docente de los programas académicos de la UNE. Reconocemos que un proceso participativo implica mayor energía y coordinaciones que un proceso centralizado y autoritario. Sin embargo, el producto suele ser más satisfactorio (Medina, 2010; Torrelles et al., 2011). 


\subsection{Participación en actividades y servicios}

Considerando el poco tiempo del que dispone el estudiantado, sus múltiples responsabilidades familiares y laborales, y su precaria situación económica, su participación en las actividades era un reto constante. Iguales situaciones han confrontado otras universidades (Bailey-Chen, 2007) y áreas de servicios de la UNE, según conversaciones informales. En el caso de la UNE, se agrava la situación ya que las actividades se ubican dentro de la estructura de Part of Term (PT). Este término académico de ocho o cinco semanas acortaba el periodo de tiempo para iniciar y concluir un programa de actividades, lo cual dificulta la participación del estudiantado, y en muchas instancias, genera "competencia" entre los cursos y otros eventos de la universidad. Concluimos con recomendaciones a considerar en el diseño e implantación de servicios dirigidos al estudiantado graduado.

\section{Recomendaciones}

Esta iniciativa evidenció que es necesaria y urgente la atención especializada al estudiantado graduado. La perspectiva con la que nos acercamos a esta población debe incluir múltiples niveles de intervención, entendiendo que les ha tocado enfrentar una realidad económica, social y laboral compleja. Ante situaciones complejas, se deben plantear respuestas creativas, innovadoras e integrales, como fue este proyecto. Afirmamos que las universidades tienen la responsabilidad ética de proveerles a sus estudiantes recursos y apoyos para que su tránsito por los estudios de posgrado sea enriquecedor y redunde en su graduación. Por lo que recomendamos en primer orden, que las IES presten mayor atención a los servicios ofrecidos a la población graduada y los alineen a las características y necesidades de este sector estudiantil.

Un tema importante que no debe pasar desapercibido, es que los servicios dirigidos a esta población no pueden perder de perspectiva el incremento de estudiantes con retos físicos y cognitivos, mujeres jefas de familia y personas desempleadas (Aponte et al., 2015; Peterson, 2006). Por tal razón, se deben contextualizar las intervenciones e integrar servicios que aborden estos asuntos enfatizando la transición al mundo laboral (Elkins, Guentzel, Gansemer-Topf, Ewing, y Turrentine, 2006). Los proyectos de esta envergadura, para garantizar el éxito, requieren del apoyo constante de la administración universitaria y de la planta docente, al igual que de otros sectores que conforman la comunidad universitaria. Estos son componentes vitales en la colaboración, apoyo y legitimación de estas iniciativas. 
El apoyo de los departamentos académicos fortalecería los ofrecimientos de servicios, e incluso, podría convertirlos en un requerimiento para todo el estudiantado. Otra recomendación sería que actividades tales como talleres de redacción y de investigación se integren al currículo desde el inicio de los estudios de posgrado. De esta manera, la participación estudiantil podría aumentar.

Es importante que las IES constantemente identifiquen ayudas económicas que le permitan al estudiantado costear sus estudios y gastos relacionados. Es imperativo identificar fuentes, como becas (provenientes de una variedad de fuentes de financiamiento) y oportunidades de internados y empleos. Los recursos económicos son una tensión constante en la vida académica del estudiantado graduado y un factor medular en sus posibilidades de concluir una carrera. Nuestra experiencia lo afirma. Por último, recomendamos que se evalúe todo esfuerzo o servicio hacia la población graduada. El elemento de evaluación debe ser constante en todo trabajo que emprendamos, así se coteja lo que funciona y se hacen los ajustes necesarios para lograr las metas. Tendremos más posibilidades de éxito si sistematizamos y evaluamos las intervenciones que realizamos. En el proyecto se evaluaba cada actividad y se rendía un informe anual a la agencia que lo financiaba. De esta forma, fuimos utilizando el insumo recibido para hacer modificaciones y reforzar acciones exitosas.

Aspiramos a que el Proyecto Título V Graduado de la UNE sirva de guía para el desarrollo de otros programas, tanto en Puerto Rico como en otros países latinoamericanos, ya que estos programas tienen un valor incalculable en el derrotero académico del estudiantado graduado. Es nuestro deseo que lo compartido en este escrito suscite reflexiones, pero, sobre todo, acciones concretas que promuevan la creación de servicios afines al perfil y las necesidades del estudiantado graduado de cada IES. Es necesario dejarle saber a cada estudiante de posgrado que es importante y que está visible ante nuestra mirada.

\section{Agradecimientos}

Agradecemos al doctor Luis Iturralde Albert por su apoyo y a la doctora Irma SerranoGarcía por su mentoría y revisiones al artículo. 


\section{Referencias}

Alicea, Dennis. (2012, marzo). Educación superior en Puerto Rico: Retos y oportunidades. Ponencia presentada en el Foro Censo 2010: Cambios socio-demográficos y su impacto en las instituciones de educación superior. Gurabo, Puerto Rico.

Aponte, Ricardo, Báez, David, Rivera, Blanca y Calderón, Jaime. (2015, mayo). Retos que enfrentan las instituciones de educación superior como resultado de los cambios sociodemográficos. Ponencia presentada en el Tercer Congreso de la Asociación de Colegios y Universidades Privadas. San Juan, Puerto Rico.

Bailey-Chen, Robin. (2007). Graduate student satisfaction with student services at a private university: Analysis of the findings base on ethnicity, gender, and race (Dissertation doctoral inedited). Pepperdine University, California, United States.

Brandes, Lisa. (2006). Graduate student centers: Building community and involving students. New Directions for Student Services, (115), 85-99. doi: 10.1002/ss.218

Cuevas, Marilis, Méndez, Nathalia y Benítez, Luis. (2014). Motivación hacia el servicio comunitario y voluntario en Puerto Rico. Ámbito de encuentros, 7(1), 47-70.

Del Rosario, Nircia. (2014). Manejo del duelo por muerte repentina e intervención con los sobrevivientes. Alianza para la Paz Social (ALAPAS), 6, 4-8.

Departamento de Comercio de los EE.UU. (2013). Puerto Rico: 2010. Resumen de características de la población y Vivienda. Washington, DC: Autor. Recuperado de https://www.census.gov/prod/cen2010/cph-1-53sp.pdf

El-Khawas, Elaine. (2003). The many dimensions of student diversity. En Susan R. Komives y Dudley B. Woodard Jr. (Eds.). Student services: A handbook for the profession (4ta ed., pp. 45-62). San Francisco, CA: Jossey-Bass.

Elkins, Becki, Guentzel, Melanie, Gansemer-Topf, Ann, Ewing, Leah, y Turrentine, Cathryn. (2006). If you want to know, ask: Assessing the needs and experiences of graduate students. New Directions for Student Services, (115), 5-17. doi: 10.1002/ss.212

Guentzel, Melanie y Elkins, Becki. (2006). Throwing pebbles at Stonehenge: Advocating for graduate and professional students. New Directions for Student Services, (115), 101106. doi: $10.1002 /$ ss.219

Haviland, Don, Shin, Seon-Hi y Turley, Steven. (2010). Now I'm ready: The impact of a professional development initiative on faculty concerns with program assessment. Innovative Higher Education, 35(4), 261-275. doi: 10.1007/s10755-010-9140-1

Luchillo, Lucas. (2010). Programas de apoyo a la formación de posgrado en América Latina: Tendencias y problemas. En Luchilo Lucas (Ed.), Formación de posgrado en América Latina: Políticas de apoyo, resultados e impacto (pp. 13-32). Buenos Aires, Argentina: Editorial Eudeba. 
Lvovich, David. (2009). Resultados e impacto de los programas de apoyo a la formación de posgrado en Argentina. Revista Iberoamericana de Ciencia, Tecnología y Sociedad, 5(13), 157-173.

Maxey, Susan. (2014). An investigation of the implementation of support services in a graduate advising center (Disertación doctoral inédita). Morehead State University, Kentucky, United States.

Medina, Maribel. (2010). Talento humano y trabajo en equipo del personal directivo de las universidades del municipio Maracaibo. Telos. Revista de Estudios Interdisciplinarios en Ciencias Sociales, 12(1), 79-97.

Patel, Vimal. (2014, marzo). Grad-school debt is said to rise rapidly and deserve more policy attention. Chronicle of Higher Education, 60(29). Recuperado de https://www.chronicle.com/article/Grad-School-Debt-Is-Said-to/145539

Peterson, Chris. (2006). Seeking balance in graduate school: A realistic expectation or a dangerous dilemma? New Directions for Student Services, (115), 31-44. doi: $10.1002 /$ ss.214

Pontious, Jason y Harper, Shaun. (2006). Principles for good practice in graduate and professional student engagement. New Directions for Student Services, (115), 47-58. doi: $10.1002 /$ ss.215

Polson, Cheryl. (2003). Adult graduate students challenge institutions to change. New directions for student services, (102), 59-68.

Puerto Rico's largest universities. (2017). Caribbean Business, 3(28), 22.

Rivera, Margarita y Calderón, Jaime. (2017). Compendio estadístico de datos sobre educación superior en Puerto Rico. San Juan, PR: Consejo de Educación de Puerto Rico.

Rivera, Christian, Toro-Alfonso, José y Díaz Meléndez, Lymari. (2013). Minorías frente al alza en la criminalidad: Percepción de seguridad de la comunidad lesbiana, gay, bisexual y transgénero (LGBT) en Puerto Rico. Revista Puertorriqueña de Psicología, 24(2), 1-14.

Rizzolo, Sonja, DeForest, Aubreena, DeCino, Daniel, Strear, Molly y Landram, Suzanne. (2016). Graduate student perceptions and experiences of professional development activities. Journal of Career Development, 43(3), 195-210. doi: $10.1177 / 0894845315587967$

Rosario, Ramón. (2014). Las condiciones laborales de los profesores en la universidad contemporánea. Kálathos, 7(2), 110-133. Recuperado de http://kalathos.metro.inter.edu/kalathos mag/publications/Las-condiciones-laborales.pdf

Rosser, Simon, Rugg, Deborah y Ross, Michael. (2001). Increasing research and evaluation productivity: Tips for successful writing retreats. Health Promotion Practice, 2(1), 9-13. doi: $10.1177 / 152483990100200103$ 
Sarason, Seymour. (1972). The creation of settings and the future societies. San Francisco, CA: Jossey-Bass.

Sistema Universitario Ana G. Méndez, Universidad del Este. (2017). Serie histórica de matrícula de agosto 2012 a agosto 2016. Documento interno sin publicar.

Skouras, Thomas. (2001). Graduate education: Needs, accommodations, and levels of service for adult students (Disertación doctoral inédita). Johnson \& Wales University, Rhode Island, United States.

Tacke, Lauren. (2005). Graduate student attitudes toward institutional student services (Disertación doctoral inédita). Dowling College, New York, United States.

Terry, Phoebe. (2002). An assessment of graduate student services at Virginia's institutions of higher education (Disertación doctoral inédita). George Washington University, Washington D.C., United States.

Torrelles, Cristina, Coiduras, Jordi, Isus, Sofía, Carreras, Xavier, París, Georgina y Cela, José. (2011). Competencia de trabajo en equipo: Definición y categorización. Profesorado. Revista de Currículum y Formación de Profesorado, 15(3), 329-344.

Universidad del Este. (2009). Manual de políticas para los programas graduados. Carolina, Puerto Rico: Autor.

Universidad del Este. (2015). Proyecto Título V Graduado. Evaluación sumativa, 2010-2015 (Informe de Evaluación). Documento interno sin publicar.

Universidad del Este. (2016). Fact sheet 2015-2016 Associate Vice Presidency of Strategic Planning and Institutional Effectiveness. [Fact sheet]. Recuperado de http://ac.suagm.edu/sites/default/files/fact sheet suagm 2015-2016.pdf

Wendler, Cathy, Bridgeman, Brent, Cline, Fred, Millet, Catherine, Rock, JoAnn, Bell, Nathan y McAllister, Patricia. (2010). The path forward: The future of graduate education in the United States. Princeton, NJ: Educational Testing Service. 